\title{
Improved spectral descriptions of planetary nebulae central stars (Research Note)
}

\author{
W. A. Weidmann ${ }^{1, \star}$, R. H. Méndez ${ }^{2}$, and R. Gamen ${ }^{3, \star \star}$ \\ 1 Observatorio Astronómico Córdoba, Universidad Nacional de Córdoba, Argentina \\ e-mail: walter@oac.uncor.edu \\ 2 Institute for Astronomy, University of Hawaii, 2680 Woodlawn Drive, HI 96822 Honolulu, USA \\ ${ }^{3}$ Instituto de Astrofísica de La Plata, CCT La Plata-CONICET, Facultad de Ciencias Astronómicas y Geofísicas Universidad \\ Nacional de La Plata, Argentina \\ e-mail: rgamen@fcaglp.unlp.edu.ar
}

Received 14 March 2015 / Accepted 2 May 2015

\begin{abstract}
Context. At least 492 central stars of Galactic planetary nebulae (CSPNs) have been assigned spectral types. Since many CSPNs are faint, these classification efforts are frequently made at low spectral resolution. However, the stellar Balmer absorption lines are contaminated with nebular emission; therefore in many cases a low-resolution spectrum does not enable the determination of the $\mathrm{H}$ abundance in the CSPN photosphere. Whether or not the photosphere is $\mathrm{H}$ deficient is arguably the most important fact we should expect to extract from the CSPN spectrum, and should be the basis for an adequate spectral classification system.

Aims. Our purpose is to provide accurate spectral classifications and contribute to the knowledge of central stars of planetary nebulae and stellar evolution.

Methods. We have obtained and studied higher quality spectra of CSPNs described in the literature as weak emission-line star (WELS). We provide descriptions of 19 CSPN spectra. These stars had been previously classified at low spectral resolution. We used medium-resolution spectra taken with the Gemini Multi-Object Spectrograph (GMOS). We provide spectral types in the Morgan-Keenan (MK) system whenever possible.

Results. Twelve stars in our sample appear to have normal $\mathrm{H}$ rich photospheric abundances, and five stars remain unclassified. The rest (two) are most probably $\mathrm{H}$ deficient. Of all central stars described by other authors as WELS, we find that at least $26 \%$ of them are, in fact, $\mathrm{H}$ rich $\mathrm{O}$ stars, and at least $3 \%$ are $\mathrm{H}$ deficient. This supports the suggestion that the denomination WELS should not be taken as a spectral type, because, as a WELS is based on low-resolution spectra, it cannot provide enough information about the photospheric $\mathrm{H}$ abundance.
\end{abstract}

Key words. planetary nebulae: general - stars: emission-line, Be - stars: evolution - stars: early-type

\section{Introduction}

Most main-sequence star spectra can be classified in a simple two-dimensional system (surface temperature and surface gravity) because they share almost the same chemical composition and do not have strong winds. When we need to allow for chemical peculiarities and strong stellar winds, the classification system necessarily becomes more complicated; that is the case for central stars of planetary nebulae (CSPNs). In particular, it is very important to decide which CSPNs are $\mathrm{H}$ deficient. This has a direct impact on the theory of stellar evolution because the simplest picture of post-asymptotic giant branch (AGB) single star evolution (e.g., Schonberner 1989) requires a departure from the AGB before the $\mathrm{H}$ rich envelope is depleted, leading to the expectation that all CSPNs retain a normal $\mathrm{H}$ rich composition. In contrast, observations clearly indicate that about $30 \%$ of CSPNs are H deficient (e.g., Mendez 1991). The most popular solution to this problem is the born-again mechanism (e.g., Iben 1984).

Hence, the empirical information about $\mathrm{H}$ deficiency is arguably the most important fact that a spectral classification for CSPNs should provide. Unfortunately, to extract this information from CSPN spectra is hard because (1) they are faint

\footnotetext{
$\star$ Member of Carrera del Investigador CONICET, Argentina.

$\star \star$ Member of Carrera del Investigador CONICET, Argentina.
}

(60\% of the CSPNs listed in the SECGPN $^{1}$ have $V>15.5$; for many other stars in that catalogue no apparent magnitude is listed); and (2) the CSPN spectra often suffer contamination from strong nebular emissions, in particular, the Balmer lines.

Because of the faintness of CSPNs, a lot of work related to spectral classification is done at low spectral resolution, but precisely because of that fact, the resulting "spectral types" frequently fail to establish whether or not the photosphere is $\mathrm{H}$ deficient. An example of this situation is the introduction by Tylenda et al. (1993) of the spectral description weak emission line stars (WELS). This denomination essentially means that the stellar emission lines are not of Wolf-Rayet type. Because of the contamination with the nebular emission spectrum at low spectral resolution, however, it is not possible to ascertain if the Balmer absorption lines are present. Some authors (e.g., Fogel et al. 2003; Werner \& Herwig 2006) have already remarked that WELS, as a group, are quite heterogeneous.

In particular, in complex cases such as CSPNs, we believe that it is important to avoid unnecessary confusion, and to restrict the terms "spectral type" and "spectral classification" to those cases where the available information permits us to decide whether or not the stellar photosphere is $\mathrm{H}$ rich. In other words, we propose to refrain from using WELS as a spectral

\footnotetext{
1 Acker et al. (1992).
} 
Table 1. GMOS spectra of WELS CSPNs.

\begin{tabular}{cllclc}
\hline \hline PN G & Name & Gemini program & Spectral range [A] & Grating & Integration [s] \\
\hline $000.3+12.2$ & IC 4634 & GS-2012A-Q-69 & $3767-5977$ & B1200 & $1 \times 900$ \\
$009.6+14.8$ & NGC 6309 & GS-2011A-Q-65 & $3767-5225$ & B1200 & $1 \times 1800$ \\
$015.4-04.5$ & M 1-53 & GS-2013A-Q-44 & $4500-5970$ & B1200 & $3 \times 390$ \\
$025.8-17.9$ & NGC 6818 & GS-2011A-Q-65 & $3770-5220$ & B1200 & $1 \times 1800$ \\
$034.6+11.8$ & NGC 6572 & GN-2014A-Q-107 & $3700-6500$ & B600 & $1 \times 1200$ \\
$038.2+12.0$ & Cn 3-1 & GN-2014A-Q-107 & $3700-6500$ & B600 & $3 \times 800$ \\
$051.9-03.8$ & M 1-73 & GN-2014B-Q-101 & $3970-5430$ & B1200 & $3 \times 450$ \\
$054.1-12.1$ & NGC 6891 & GN-2014A-Q-107 & $3700-6500$ & B600 & $3 \times 600$ \\
$057.2-08.9$ & NGC 6879 & GN-2014B-Q-101 & $3970-5430$ & B1200 & $3 \times 500$ \\
$058.3-10.9$ & IC 4997 & GN-2014A-Q-107 & $3700-6500$ & B600 & $3 \times 1200$ \\
$096.4+29.9$ & NGC 6543 & GN-2014A-Q-107 & $3700-6500$ & B600 & $3 \times 200$ \\
$159.0-15.1$ & IC 351 & GN-2014B-Q-101 & $3970-5430$ & B1200 & $3 \times 900$ \\
$190.3-17.7$ & J 320 & GN-2014B-Q-101 & $3970-5430$ & B1200 & $3 \times 400$ \\
$307.2-09.0$ & He 2-97 & GS-2013A-Q-44 & $4500-5970$ & B1200 & $2 \times 210$ \\
$312.3+10.5$ & NGC 5307 & GS-2011A-Q-65 & $3767-5228$ & B1200 & $1 \times 900$ \\
$345.0-04.9$ & Cn 1-3 & GS-2013A-Q-44 & $4500-5970$ & B1200 & $2 \times 340$ \\
$348.0-13.8$ & IC 4699 & GS-2013A-Q-44 & $4500-5970$ & B1200 & $3 \times 410$ \\
$349.3-01.1$ & NGC 6337 & GS-2011A-Q-91 & $4000-5520$ & B1200 & $4 \times 600$ \\
$355.7-03.5$ & H 1-35 & GS-2013A-Q-44 & $4500-5970$ & B1200 & $2 \times 760$ \\
\hline
\end{tabular}

type, because it induces us to think that all the stars so described have similar spectra, and that may not be the case.

Of course this statement can be tested empirically, which is the purpose of this paper. Of at least 492 CSPNs that have been spectroscopically described, 72 have been called WELS (Weidmann \& Gamen 2011b). We selected a sample of 19 WELS CSPNs ( $26 \%$ of the total), and we endeavoured to obtain higher resolution spectra, which allows us to qualitatively determine if their photospheres are $\mathrm{H}$ rich or $\mathrm{H}$ deficient.

Section 2 describes the spectrograms and reduction procedures, Sect. 3 explains the classification criteria, and Sect. 4 presents the spectral descriptions and classifications. We present and summarize our conclusions in Sects. 5 and 6.

\section{Observations and data reduction}

We observed nineteen CSPN described as WELS with Gemini Multi-Object Spectrographs (GMOS-N and GMOS-S) at the Gemini Telescopes North and South, see Table 1. We ordered these objects by Galactic longitude.

Our spectrograms were acquired under programs GS2013A-Q-44, GN-2014A-Q-107 and GN-2014B-Q-101 (PI: Weidmann). We increased our sample with all GMOS CSPN spectra that were publicly available in the Gemini database.

For our programs, we selected the $\mathrm{B} 1200$ grating $(R \simeq 2300)$ at long-slit mode used with a $0.75^{\prime \prime}$ slit, or grating B600 $(R \simeq$ 1700 ) with a $1^{\prime \prime}$ slit. The selected spectral range (see Table 1) is useful to identify/classify O-type as well as Wolf-Rayet stars.

We chose the science exposure times as a function of the CSPN magnitude (Acker et al. 1990) to reach a signal-to-noise up to 100 (see Table 1). The strong nebular emission lines frequently saturated the CCD chip.

To reduce the data, we followed standard procedures using tasks in the gemini.gmos IRAF package as well as generic IRAF $^{2}$ tasks. The reduction procedures included image trimming, flat fielding, wavelength calibration, sky subtraction, and

\footnotetext{
2 IRAF is distributed by the National Optical Astronomical Observatories, operated by the Association of Universities for Research in Astronomy, Inc., under contract to the National Science Foundation of the USA.
}

relative flux calibration (the spectrophotometric standard star EG 131 was used for this purpose, see Bessell 1999).

The spectra were reduced separately and then combined for the final 1D-spectral extraction. We rectified the continua, and shifted the spectra in wavelength to zero radial velocity. Whenever possible, the contribution from the nebula was subtracted by interpolating nebular regions on opposite sides of the CSPN.

\section{Spectral classification criteria}

Given the high surface temperatures of CSPNs, we clearly have to deal with the earliest spectral types in the MK system. The MK system has recently been extended (Sota et al. 2011, 2014), building on earlier work by Nolan Walborn. See also Walborn et al. (2010). It should be obvious that only some CSPN spectra can be given an MK type. The MK system presupposes a normal $\mathrm{H}$ rich photospheric composition and low or, at most, moderate mass-loss rate. If any of these conditions are not met, each individual spectrum becomes almost unique, and the exercise of spectral classification becomes much harder.

We decided to proceed in the following way. For H rich stars classificable as $\mathrm{O}$ and $\mathrm{Of}$, we tried to provide the best possible classification in the system of Sota et al. (2011). Note, in particular, the list of qualifiers in their Table 3. It should be clear that using the Sota et al. spectral types necessarily implies that the star in question is demonstrably $\mathrm{H}$ rich. The "luminosity classes" should instead be interpreted as "surface gravity classes"; since CSPNs are less massive, they are of course less luminous than the typical massive O-type stars of Sota et al. with similar luminosity classification.

Among CSPNs we also find spectra that are quite different from any MK standard. In these cases, we prefer to follow the classification scheme of Mendez (1991). In particular, we follow the spectral type Of-WR(H), indicating a denser stellar wind, with broad emission lines and blue-shifted profiles for key diagnostic lines, plus essentially normal $\mathrm{H}$ rich composition and the spectral type $\mathrm{O}(\mathrm{He})$, which indicates a $\mathrm{H}$ poor photosphere with $\mathrm{He}$ as the predominant element. Our identification of $\mathrm{H}$ or $\mathrm{He}$ as the most abundant photospheric element is based on the behavior of the intensities of even-n and odd-n Pickering 
W. A. Weidmann et al.: Improved spectral descriptions of planetary nebulae central stars $(R N)$

Table 2. Improved spectral classification and key lines in medium resolution spectra of WELS CSPNs.

\begin{tabular}{|c|c|c|c|c|c|c|c|c|c|c|}
\hline PN G & Name & S.T. & 4542 & 4686 & 5412 & $\mathrm{H} \beta$ & $\mathrm{N} \mathrm{v}$ & CIV & $\mathrm{OV}$ & N.S. \\
\hline $000.3+12.2$ & IC 4634 & O3 If* & $\mathrm{A}$ & $\mathrm{AE}$ & $\mathrm{A}$ & $\mathrm{A}$ & $\mathrm{A}$ & $E$ & $\mathrm{~A}$ & $\mathrm{y}$ \\
\hline $009.6+14.8$ & NGC 6309 & $\mathrm{O}(\mathrm{He})$ & A & A & A? & $\mathrm{A}$ & $\mathrm{E}$ & $\mathrm{E} ?$ & - & $\mathrm{y}$ \\
\hline $015.4-04.5$ & M 1-53 & $\mathrm{O} 3 \mathrm{I} \mathrm{f}$ & A & $\mathrm{E}$ & A & A & A & $\mathrm{E}$ & A & $\mathrm{y}$ \\
\hline $025.8-17.9$ & NGC 6818 & not classif & $\mathrm{A}$ ? & $\mathrm{E}$ & A? & $\mathrm{A}$ ? & - & $\mathrm{E}$ & - & $\mathrm{y}$ \\
\hline $034.6+11.8$ & NGC 6572 & Of-WR(H) & A & $\mathrm{E}$ & $\mathrm{E}$ & $\mathrm{E}$ & $\mathrm{E}$ & E & A & $\mathrm{y}$ \\
\hline $038.2+12.0$ & Cn 3-1 & $\mathrm{O} 7 \mathrm{Ib}(\mathrm{f})$ & A & - & A & A & - & A & - & $\mathrm{n}$ \\
\hline $051.9-03.8$ & M 1-73 & O3.5 I f & A & E & A & $\mathrm{AE}$ & A & E & - & $\mathrm{y}$ \\
\hline $054.1-12.1$ & NGC 6891 & O3 Ib (f*) & A & $\mathrm{AE}$ & A & A & A & E & A & $\mathrm{y}$ \\
\hline $057.2-08.9$ & NGC 6879 & $\mathrm{O} 3 \mathrm{f}(\mathrm{He})$ & A & $\mathrm{E}$ & A & $\mathrm{A}$ ? & A & $\mathrm{E} ?$ & A & $\mathrm{n}$ \\
\hline $058.3-10.9$ & IC 4997 & not classif & A & $\mathrm{E}$ & - & - & $\mathrm{A} ?$ & $\mathrm{E}$ & - & $\mathrm{n}$ \\
\hline $096.4+29.9$ & NGC 6543 & Of-WR(H) & $\mathrm{A}$ ? & E & $\mathrm{E}$ & E & $\mathrm{E}$ & E & A & $\mathrm{y}$ \\
\hline $159.0-15.1$ & IC 351 & $\mathrm{Of}(\mathrm{H})$ & A & $\mathrm{E}$ ? & A & A & $\mathrm{E}$ & $\mathrm{N} / \mathrm{D}$ & A? & $\mathrm{n}$ \\
\hline $190.3-17.7$ & J 320 & O3 V ((f)) & A & A & A & A & A & $\mathrm{N} / \mathrm{D}$ & A & $\mathrm{y}$ \\
\hline $307.2-09.0$ & He 2-97 & Of-WR(H) & $\mathrm{AE}$ & $\mathrm{E}$ & - & $\mathrm{E}$ & $\mathrm{E}$ & $\mathrm{E}$ & - & $\mathrm{n}$ \\
\hline $312.3+10.5$ & NGC 5307 & $03.5 \mathrm{~V}$ & A & A & A & $\mathrm{A}$ & A & $\mathrm{E}$ & A & $\mathrm{y}$ \\
\hline $345.0-04.9$ & Cn 1-3 & not classif & A & E? & A & E? & - & E & - & $\mathrm{n}$ \\
\hline $348.0-13.8$ & IC 4699 & O3 V ((f)) & A & A & A & A & A & E & A & $\mathrm{y}$ \\
\hline $349.3-01.1$ & NGC 6337 & not classif & $\mathrm{E}$ & $\mathrm{E}$ & $\mathrm{E}$ & $\mathrm{E}$ & $\mathrm{E}$ & E & - & $\mathrm{y}$ \\
\hline $355.7-03.5$ & H 1-35 & not classif & A & $\mathrm{E}$ ? & A? & E & $\mathrm{E} ?$ & $\mathrm{E} ?$ & $\mathrm{E} ?$ & $\mathrm{n}$ \\
\hline
\end{tabular}

Notes. The N.S. column indicates whether or not it was possible to subtract the nebular component. The letters A and E mean that the ion was in absorption and emission, respectively. An undetected ion was denoted (-), and N/D means no data available in the corresponding spectral range. The columns labeled "N V", "C IV" and "O V" indicate the doublet at 4603-19 $\AA$, the doublet at 5801-11 $\AA$ and $5114 \AA$ respectively. References for the C IV doublet at $5806 \AA$ are Weidmann \& Gamen (2011a), Tylenda et al. (1993), and the spectra shown in Figs. 6.

Table 3. Other O- and Of-type CSPNs described as WELS.

\begin{tabular}{clccc}
\hline \hline PN G & Name & S.T. & References & CIV emission ref. \\
\hline $002.0-06.2$ & M 2-33 & O5f(H) & HP2007 & HP2007 \\
$002.1-02.2$ & M 3-20 & Of & AK1987 & em? GC2009 \\
$003.9-14.9$ & Hb 7 & O3 & GP2001 & no em TA1993 \\
$016.4-01.9$ & M 1-46 & Of(H) & H2003 & no em TA1993 \\
$264.4-12.7$ & He 2-5 & Of(H) & M1991 & N/D \\
$315.1-13.0$ & He 2-131 & O8(f)p & MN1979 & no em TA1993 \\
$316.1+08.4$ & He 2-108 & Of(H) & M1991 & PCygni MA2003 \\
\hline
\end{tabular}

References. HP2007 (Hultzsch et al. 2007), H2003 (Handler 2003), GP2001 (Gauba et al. 2001), M1991 (Mendez 1991), AK1987 (Aller \& Keyes 1987), MN1979 (Mendez \& Niemela 1979). GC2009 (Górny et al. 2009), TA1993 (Tylenda et al. 1993), MA2003 (Marcolino \& de Araújo 2003).

He II lines; the even-n lines blend with the corresponding Balmer lines.

The determined spectral types are given in Table 2, and the spectra are shown in Figs. 1 to 5, arranged according to the spectral type. In addition, we include the red part of the spectra (Figs. 6) to highlight the presence and intensity of ions such as $\mathrm{O} v$ and $\mathrm{CIV}$.

In next section, we present individual descriptions for each of the central stars we observed. In some cases, the strength of the nebular emission lines made classification impossible ( $\mathrm{H}$ abundance undecided). In these cases the only solution is higher spectral resolution and/or much more efficient nebular subtraction, which can be obtained, for example, in excellent seeing conditions using a much narrower spectrograph slit.

\section{Stellar descriptions and classifications: notes on individual objects}

IC 4634: Balmer and He II absorption lines clearly visible. The $4686 \AA$ emission line shows a P Cygni-type profile, obviously of stellar origin. Together with a N IV $4058 \AA$ emission stronger than N III $4640 \AA$ emission, this indicates a qualifier f*. The
$\mathrm{N} \mathrm{V}$ absorption doublet is strong, but weaker than He II 4541, leading to a spectral type $\mathrm{O} 3$.

NGC 6309: He II 4686 and 4541 in absorption. Since 4541 appears to be stronger than any absorption at 4340 , we believe that this star is a new example of spectral type $\mathrm{O}(\mathrm{He})$ (Mendez 1991).

M 1-53: Balmer and He II 4541 in absorption. The N v 4603-19 $\AA$ absorption doublet is present, but weaker than 4541, indicating a spectral type O3. Other lines present: N III 4634-40-42 ̊, C IV $4658 \AA$ emissions, O v $5114 \AA$ absorption. The presence of O VI emission at $5290 \AA$ further suggests a very hot star.

NGC 6818: this nebula's angular size made a reasonable nebular emission subtraction possible. However, the nebular emissions are so strong that no stellar feature is clearly visible, with the only exception of He II 4686 emission which we could not classify. Higher spectral resolution will be necessary.

NGC 6572: broad Balmer and He II 4686 emissions (most absorptions seen in our spectrum are an artifact of nebular subtraction). Note that 4541 is not in emission, therefore this star cannot be classified as a Wolf-Rayet. There may be a stellar absorption of C III at $4069 \AA$. There is strong C III emission 
A\&A 579, A86 (2015)
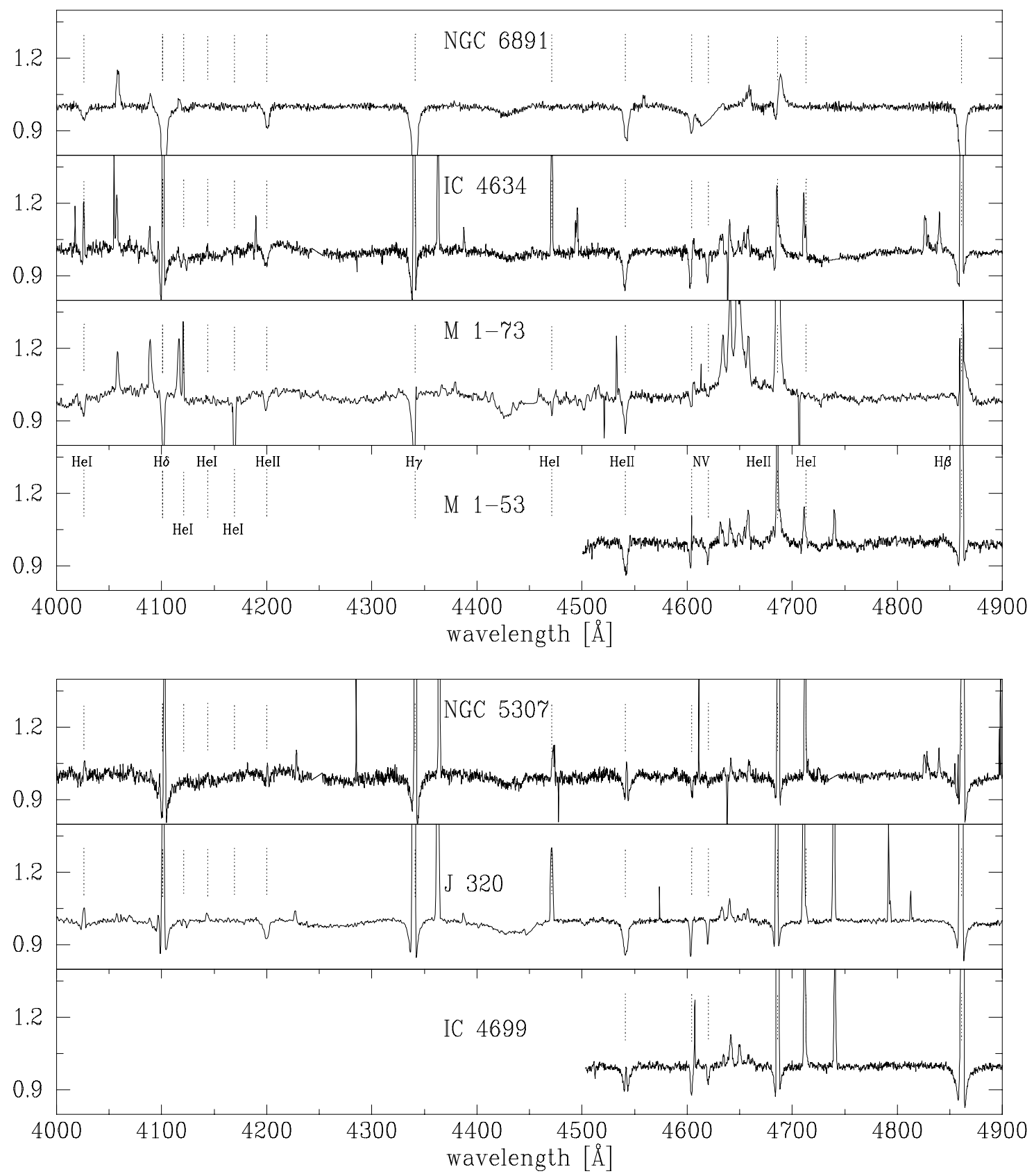

Fig. 1. Normalized spectra of O-type CSPN grouped according to their spectral classification: O3I and O3V (see Table 1). The interstellar absorption band at $\lambda 4428$ are not indicated. The most important spectral features (absorption or emission) identified are $\mathrm{H} \beta, \mathrm{H} \gamma$, and $\mathrm{H} \delta$. $\mathrm{He} \mathrm{I} \lambda 4026$, 4121, 4144, 4169, 4471, and 4713. He II $\lambda 4200,4541$, and 4686. N v $\lambda 4604-19$.

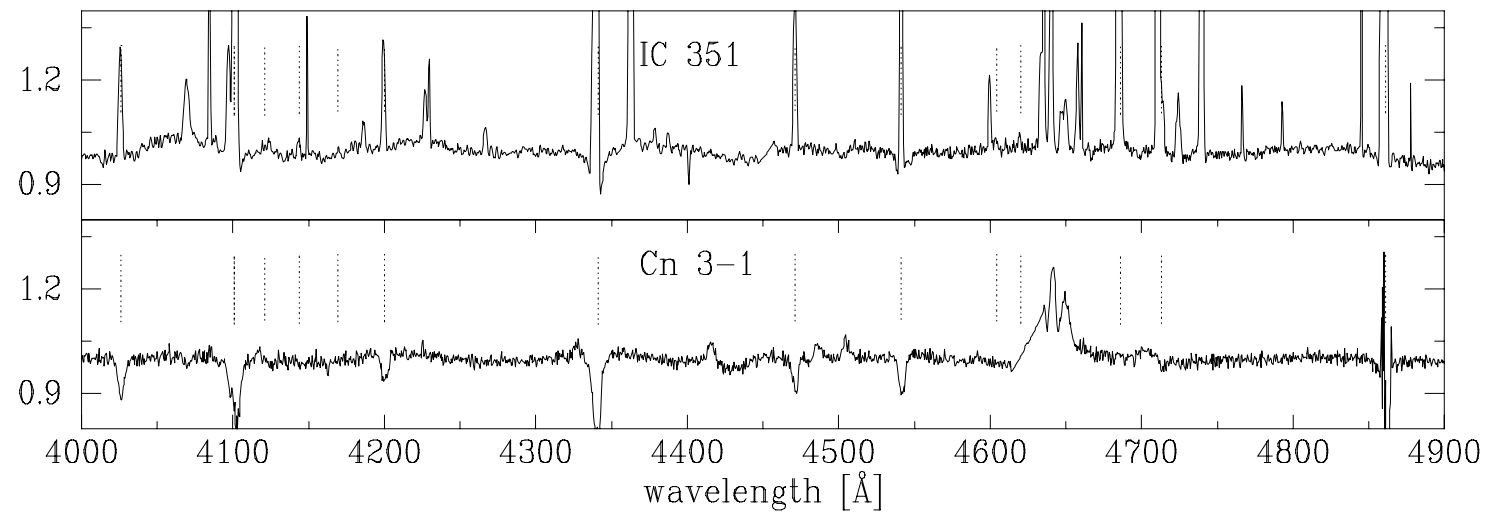

Fig. 2. Normalized spectra of early-O CSPN. 
W. A. Weidmann et al.: Improved spectral descriptions of planetary nebulae central stars $(R N)$

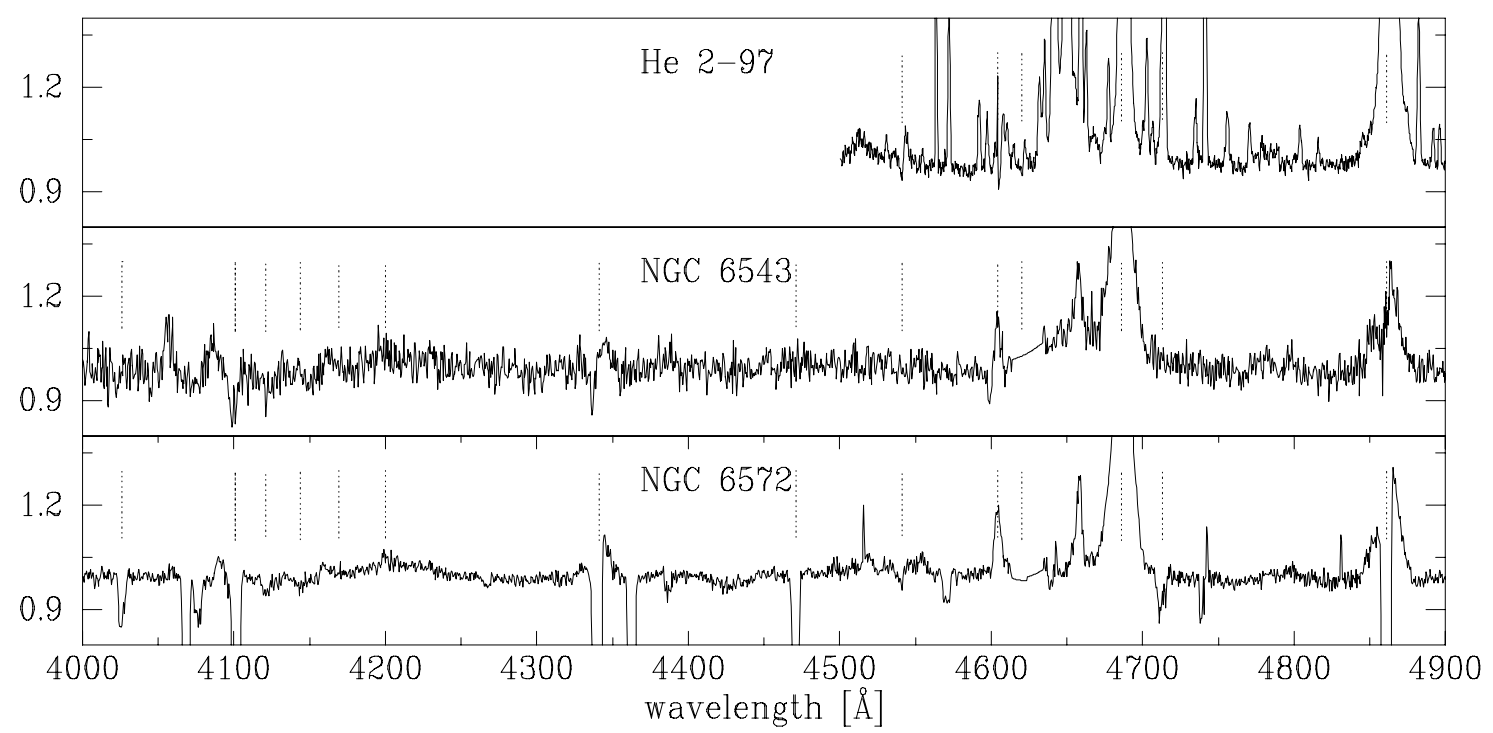

Fig. 3. Normalized spectra of Of-WR(H) CSPN. Note the broad emission of He II at $4686 \AA$.

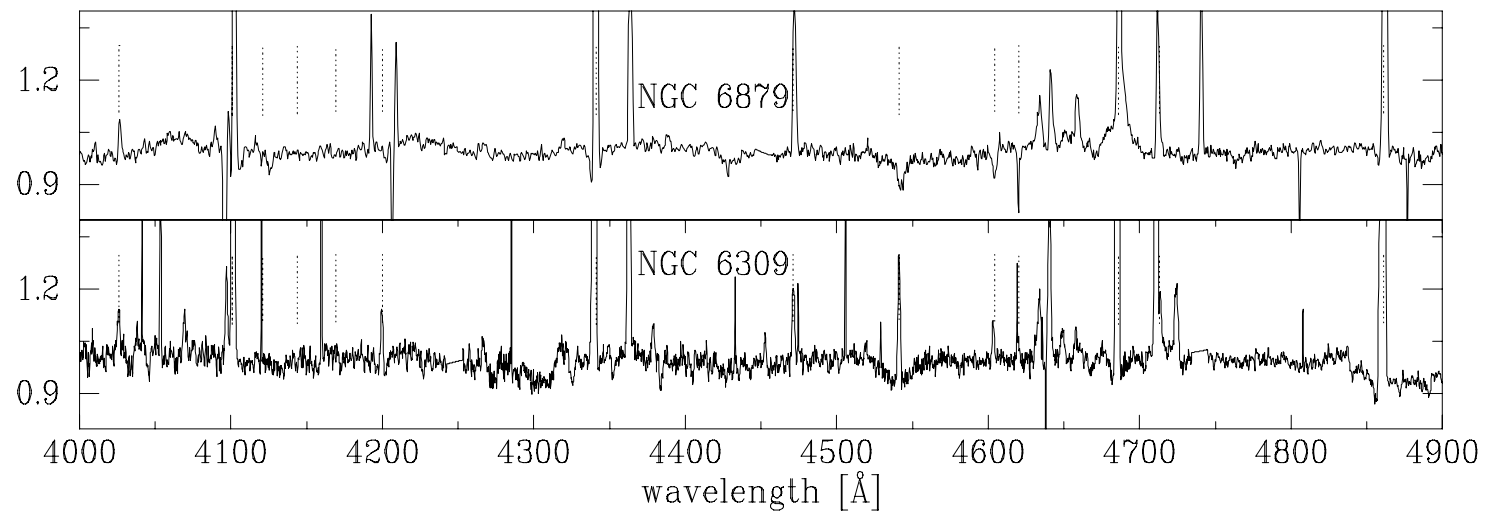

Fig. 4. Normalized spectra of possible $\mathrm{O}(\mathrm{He}) \mathrm{CSPN}$.

at $4658 \AA$. O VI emission is visible at $5290 \AA$. This star has been classified as Of-WR(H) by Mendez (1991). A good spectrum longward of $4200 \AA$ has been published by Mendez et al. (1990).

Cn 3-1: Balmer lines in absorption. Both He I 4471 and He II 4541 clearly seen in absorption; this indicates a rather lowtemperature star. From the ratio $4471 / 4541$ it is an $\mathrm{O} 7$ star. The C IV doublet at $5806 \AA$ is in absorption, again consistent with a lower temperature (this doublet changes from absorption into emission at effective temperatures around $50000 \mathrm{~K}$; see Sect. 5.1 below). There is absorption of C III and N III at 4070 and $4097 \AA$, respectively, together with Si IV at 4089 and $4116 \AA$. Since there is emission at N III 4634-40-42 $\AA$, and He II 4686 is absent, presumably filled with emission, we adopt a qualifier (f) for this star. Note the presence of S IV emission at 4486, $4504 \AA$. Another nice example of this S IV doublet in a central star spectrum is shown in Fig. 1 of Hultzsch et al. (2007).

M 1-73: the Balmer profiles are affected by nebular subtraction, but $\mathrm{H} \beta$ appears to show a $\mathrm{P}$ Cygni profile. Well-defined absorption lines of He II (at 5412, 4541 and $4200 \AA$ ). The He II stellar feature at 4686 has an equivalent width of $4.3 \AA$, suggesting photospheric emission (the nebular FWHM is $2.2 \AA$ ), therefore we classify this star as Of. The absorption lines of N v suggest an $\mathrm{O} 3.5$ star.
NGC 6891: a reasonably good nebular emission subtraction was possible. The Balmer series and He II absorptions are clearly visible. He II at $4686 \AA$ shows a P Cygni profile. The N V absorption at $4603 \AA$ is clearly present (the 4619 line is not visible because of a CCD gap). We adopt a type O3. The weakness of N III at $4640 \AA$ and the prominent emission of N IV at $4058 \AA$ suggests a qualifier (f*). Si IV at 4088, $4116 \AA$, and C IV at $4658 \AA$ appear in emission. This CSPN was classified as Of(H) (Mendez 1991), essentially in agreement with our classification.

NGC 6879: compact nebula, very strong nebular emissions. Since the absorption at He II 4541 appears to be slightly stronger than the absorption at 4340 , we believe that this star may be $\mathrm{H}$ deficient, although a higher resolution spectrum would be desirable. The presence of N v 4603-19 indicates an early spectral type. Since N III 4634-40-41, and He II 4686 are in emission, we adopt a spectral type O3f(He), following Mendez (1991). Si IV 4088, 4116 appear to be in emission.

IC 4997: this nebula is spatially unresolved, and the stellar spectrum is severely contaminated with nebular emissions. We see absorption lines of He II (4542, 4200 and $5412 \AA$ ). The presence of photospheric $\mathrm{H}$ cannot be decided. The emission of He II at $4686 \AA$ is wider than nebular lines, suggesting a stellar origin.

$N G C$ 6543: this star has been classified as Of-WR(H) by Mendez (1991), indicating that it is H rich. The spectrum is similar to that of NGC 6572. Georgiev et al. (2008) analyzed a high 

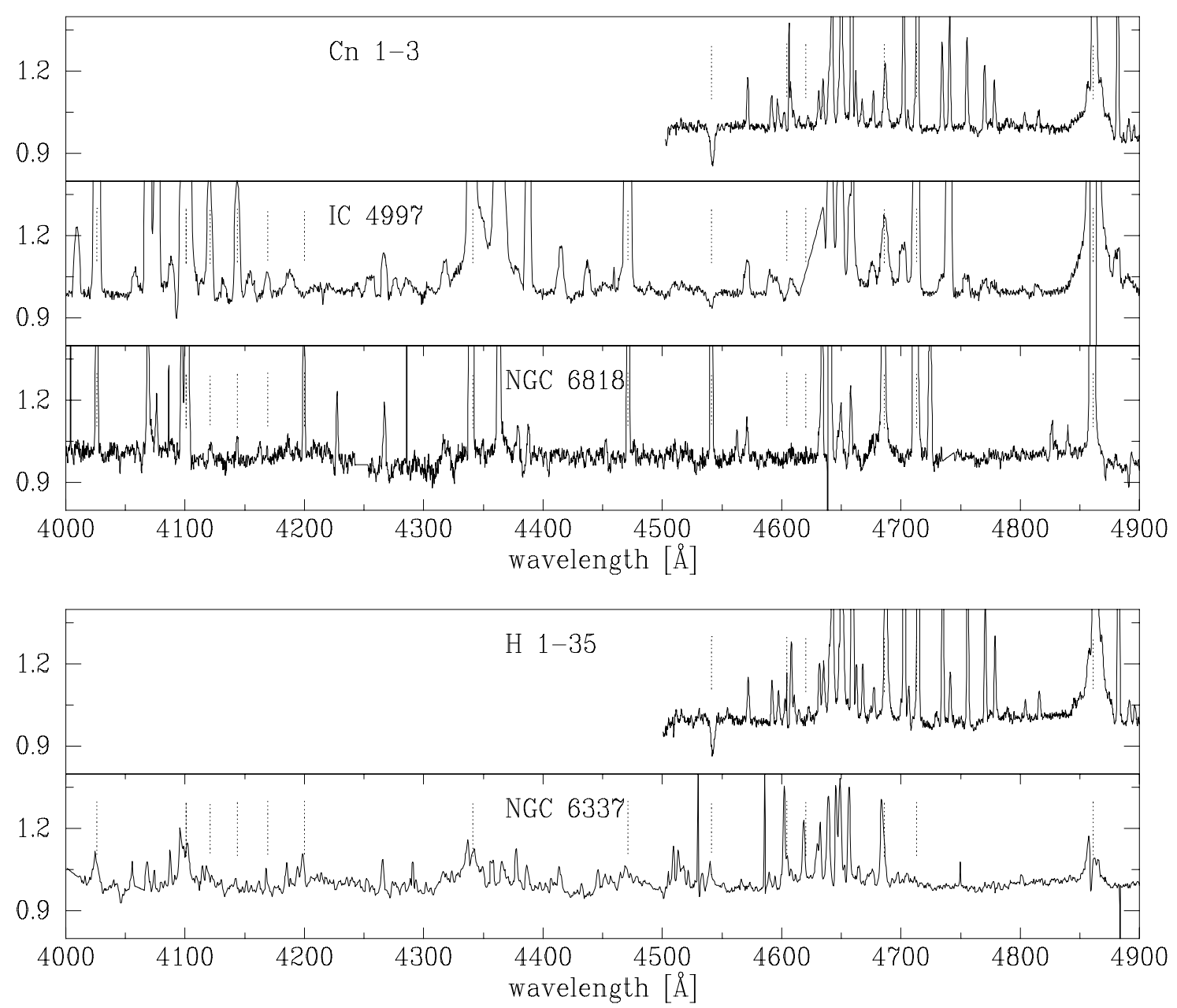

Fig. 5. CSPN without classification. Note the evident absorption of He II in Cn 1-3, IC 4997, and H 1-35.

quality spectrum of this star. Although they did not propose any spectral classification, they confirmed that the star is not $\mathrm{H}$ poor. Our spectral range allows us to describe C III at $5696 \AA$ and C IV at $5806 \AA$. The former is absent, but the C IV emission is very intense and broad, confirming the impression that this star's wind is somewhat intermediate between Of and Wolf-Rayet, in agreement with (Mendez 1991). Other emission lines we identified are N IV at $4058 \AA$ and N V at $4603 \AA, C$ IV at $4658 \AA$, He II at 4686 and $5412 \AA$, O VI at $5290 \AA$.

IC 351: the stellar spectrum shows clear evidence of $\mathrm{H} \gamma 4340$ and He II 4541, both in absorption. The He I line at $4471 \AA$ is not visible because of nebular contamination, so we cannot estimate a reliable $\mathrm{O}$ subtype. This star is probably Of, although we cannot decide if there is any He II stellar emission at $4686 \AA$.

$J$ 320: this object shows a clearly defined set of $\mathrm{H}$ and He II absorption lines, which together with strong N V 4603-19 absorptions and weak N III 4634-40-41 emissions is consistent with a spectral type $\mathrm{O} 3((\mathrm{f}))$.

He 2-97: this nebula is not resolved and the nebular lines are quite strong, however, part of the $\mathrm{H} \beta$ emission appears to be of stellar origin. The emissions of He II and C IV at 4686 and 5801-12 $\AA$ are wide (FWHM 6.5, 5.5 and 5.9 $\AA$, respectively, while nebular lines have a FWHM of $2.0 \AA$ ). An interesting feature is the P Cygni profile of He II at $4541 \AA$, indicating a dense stellar wind. This central star looks similar to those of NGC 6543 and NGC 6572, but with stronger C III 4650. Following Mendez (1991), we have adopted a type Of-WR(H).

NGC 5307: the spectrum shows a clear Balmer series and He II lines all in absorption, in particular at $4686 \AA$. Absorption of N V at $4603 \AA$ is visible. In addition, weak C IV emission at $4658 \AA$ is visible. We adopt a spectral type O3.5 V.

Cn 1-3: the only clear absorption we see is He II 4541. The emission at $4686 \AA$ is wider than the nebular lines, suggesting a stellar component and therefore a spectral type Of. It is not clear if $\mathrm{H} \beta$ has a stellar emission component, and therefore we refrain from classifying this star.

IC 4699: the spectrum shows a clear Balmer series and He II lines all in absorption, in particular at $4686 \AA$. Absorptions of $\mathrm{N} \mathrm{V}$ at $4603,19 \AA$ are visible. In addition, weak N III emissions at $4634,40,41 \AA$ are visible. We adopt a spectral type $\mathrm{O} 3 \mathrm{~V}((\mathrm{f}))$.

NGC 6337: this central star is a binary (Hillwig et al. 2006), detected through photometric variability. The emission lines are probably due to the irradiated companion (De Marco et al. 2008). No spectrum of this star has been published previously. Our spectrum looks similar to that of the central star of IPHASXJ194359.5+170901 (Corradi et al. 2011). There is no clear absorption line, however, we see a combination of absorption and emission lines at the Balmer series. This is confirmed by spectra taken at CASLEO (El Leoncito Astronomical Complex, San Juan, Argentina), with the $215 \mathrm{~cm}$ Sahade telescope; these 
W. A. Weidmann et al.: Improved spectral descriptions of planetary nebulae central stars $(R N)$
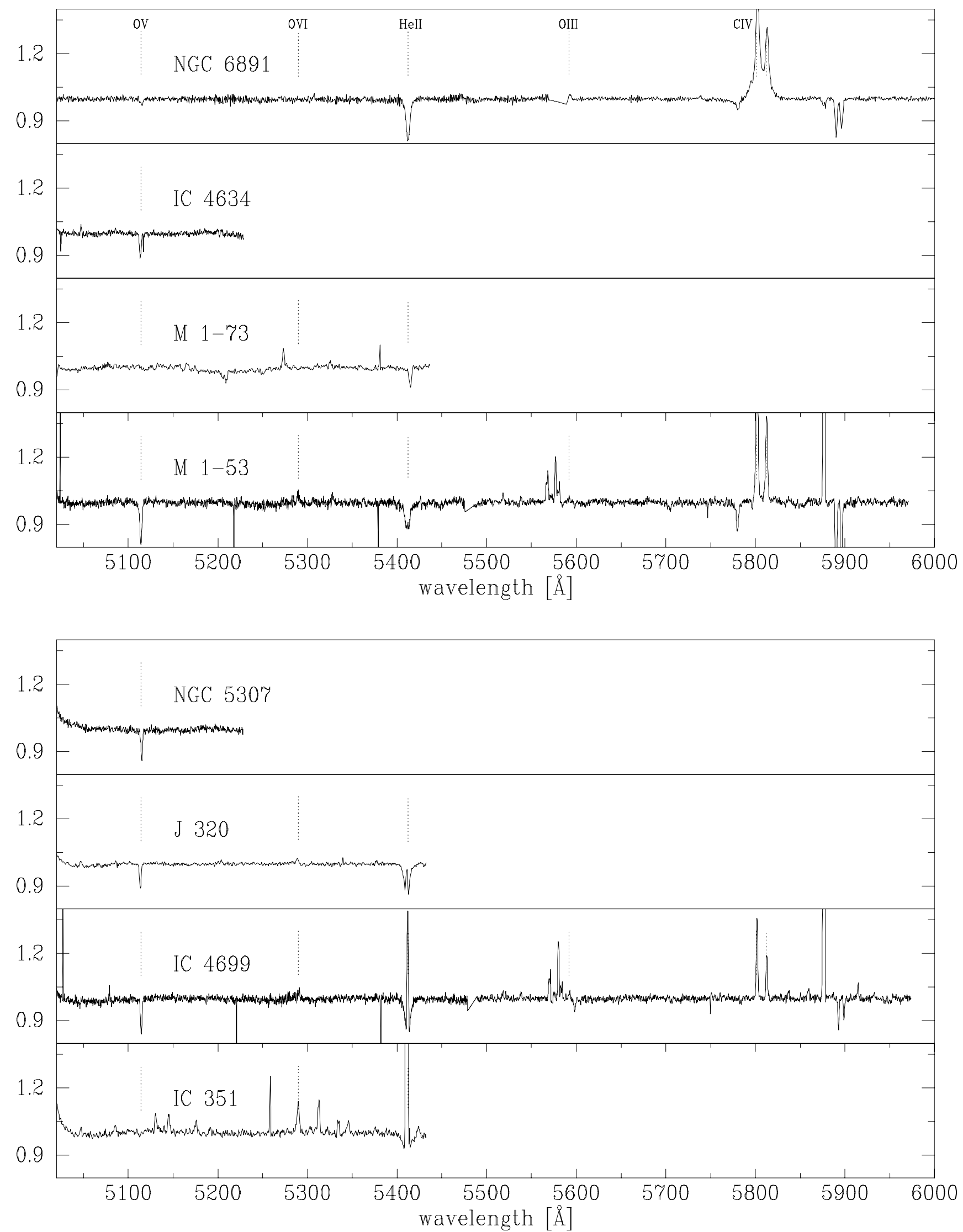

Fig. 6. "Red" part of the spectra shown in Figs. 1 to 5 . Note the presence of the ions O V at $5114 \AA$, O VI at $5290 \AA$, O III at $5592 \AA$, He II at $5412 \AA$ and C IV at $5806 \AA$. 

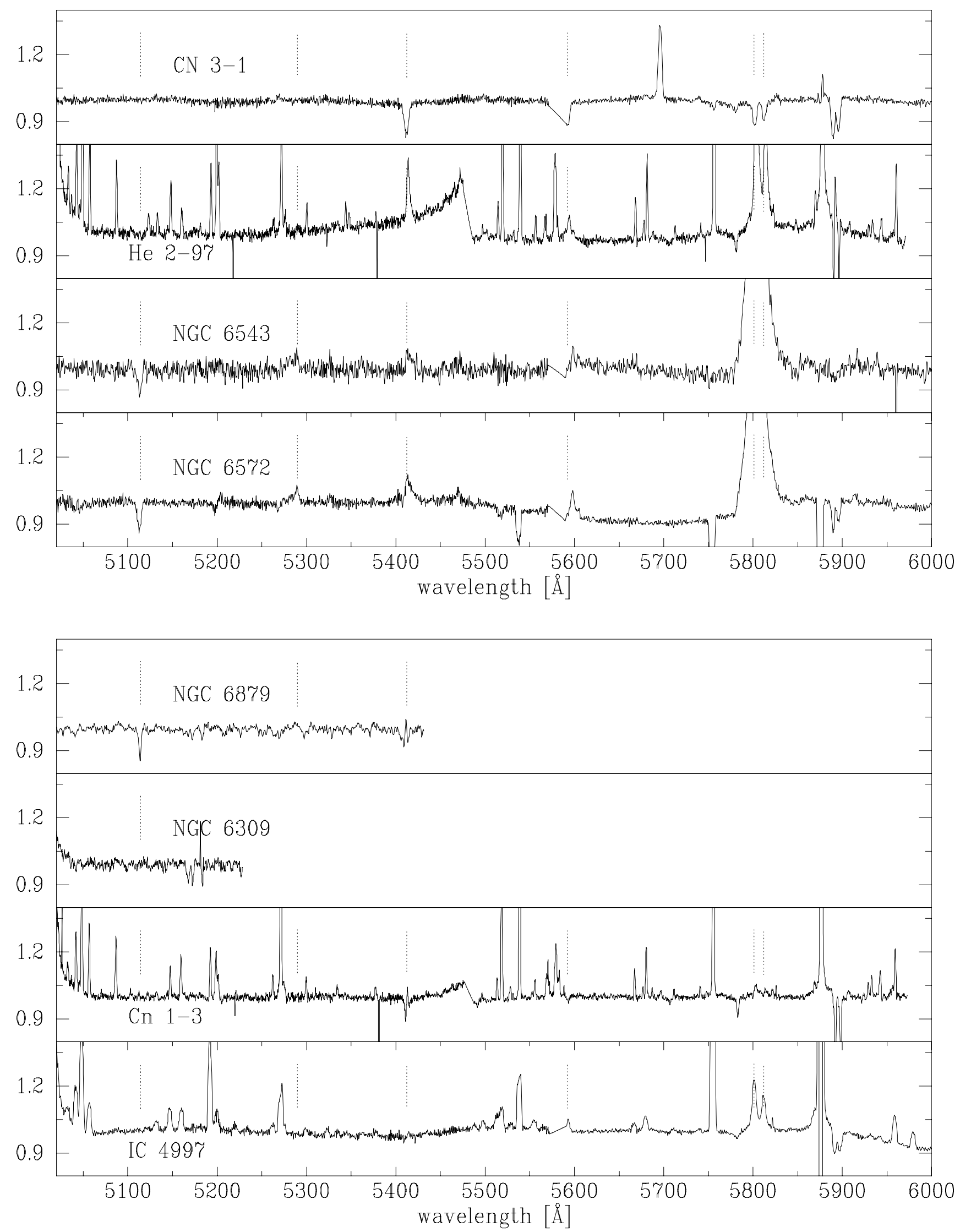

Fig. 6. continued. 


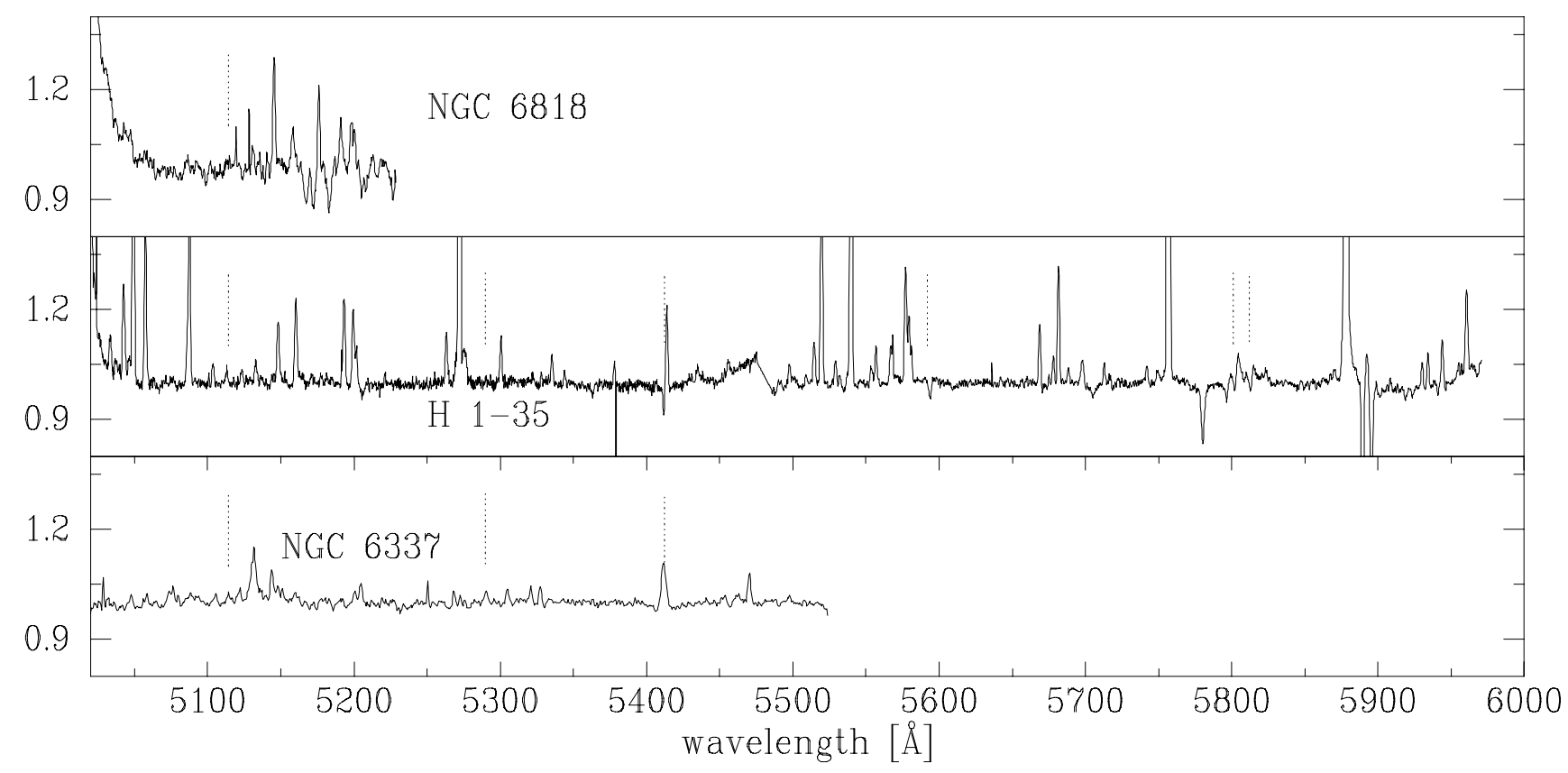

Fig. 6. continued.

spectra display a strong $\mathrm{H} \alpha$ absorption. This suggests that the brightest star in the binary system is not a late-type star, and that it is $\mathrm{H}$ rich, but we refrain from classifying this star. There is an emission blend near 4650 A, i.e., N v 4603-19, N III 4634-41 A, C III 4647-51 ̊, C IV $4658 \AA$, and He II $4686 \AA$. This collection of emission lines led to describe this star as WELS.

$H$ 1-35: the available section of the spectrum is very similar to that of $\mathrm{Cn} \mathrm{1-3.} \mathrm{There} \mathrm{seems} \mathrm{to} \mathrm{be} \mathrm{a} \mathrm{stellar} \mathrm{H} \beta$ emission component, and the only absorption feature visible is He II 4541 . We refrain from classifying this star.

\section{Discussion and interpretation of our classifications}

The WELS denomination was introduced by Tylenda et al. (1993). It has been used when some of the following stellar emission lines are observed: the 4650 group (N III $4634+$ $\mathrm{N}$ III $4641+\mathrm{C}$ III 4647 + C III 4650 + C IV 4658), He II 4686, and C IV 5801-11. We explained in the introduction why we think this denomination should not be used as a spectral type. Other authors have expressed similar reservations: Miszalski (2012) and Kwitter et al. (2014, Sect. 5.3). We now summarize the result of our empirical test.

Our spectroscopic survey of 19 WELS stars has indeed shown a variety of spectral types. Nine CSPNs turn out to be $\mathrm{H}$ rich $\mathrm{O}$ and $\mathrm{Of}$ stars. Three can be classified as Of-WR(H) stars, in view of their denser stellar winds (Mendez 1991). In fact, two of them (NGC 6543 and NGC 6572) had been previously described as $\mathrm{H}$ rich, based on high-resolution spectra, before being described as WELS. We did not find any Wolf-Rayet star.

Hence, in our sample of 19 CSPNs, we found a total of $12 \mathrm{H}$ rich stars. We classified two stars as probably $\mathrm{O}(\mathrm{He})$, although higher resolution spectra would be desirable to provide better confirmation. Five stars could not be reliably classified, again requiring a higher spectral resolution. We confirm that the denomination WELS means, in practice, "insufficient spectral information" to decide if these stars are $\mathrm{H}$ rich or not. Most of the WELS stars turn out to be $\mathrm{H}$ rich.
For completeness, it may be useful to add (see Table 3) a list of CSPNs, described as WELS from low-resolution spectra, that are in fact $\mathrm{O}$ and $\mathrm{O}$ H rich stars, previously and/or subsequently classified as such on the basis of better spectrograms.

\subsection{The CIV emission}

A common feature in stars described as WELS is the emission doublet of CIV at 5801-11 $\AA$. It is commonly attributed to the CSPN, but in some cases it may be due to the nebula instead; see the discussion of the stellar spectrum of NGC 5979 in Górny (2014). Clearly, if the PN is spatially unresolved and of high excitation, it may be hard to decide its origin. A higher spectral resolution would be required, for example to verify if a double-peaked emission due to nebular expansion can be resolved.

However, in many CSPNs this C IV doublet is clearly of stellar origin. We compare now this C IV emission with Pop I stars. According to Conti (1974), the C IV doublet is not seen in emission, even in the most extreme Of stars. Jaschek \& Jaschek (1995) agrees, also noting that O-type stars show this doublet in absorption, with a maximum around O7. Walborn (2001) recognizes that the emission of $\mathrm{CIV}$ at $5806 \AA$ has not received too much attention in the literature. In addition, C IV emission is not reported in sdO stars (Drilling et al. 2013).

Walborn \& Howarth (2000) have shown that these C IV lines are visible in emission in the spectrum of $\mathrm{O} 3$ If* type stars, almost neutral in $\mathrm{O} 4 \mathrm{If}$, and as pure absorption lines in O5 If type star. Moreover, they are also in emission in the spectrum of the O2 I star HD 93129A.

In summary, these narrow emission lines are common in the O-type stars that are nuclei of planetary nebulae but not in those of Population I. The difference could be a surface temperature effect. Indeed, CSPNs with $T_{\text {eff }}<45000 \mathrm{~K}$ always display C IV at $5806 \AA$ in absorption (e.g., He 2-138, M 1-26, Tc 1, IC 418). On the other hand, CSPNs with $T_{\text {eff }}>50000 \mathrm{~K}$ usually present 5801-11 in emission (e.g., NGC 1535, NGC 3242, NGC 4361, NGC 7009). As we described, in Pop I O-type 
stars these emissions are only detected in the spectra of $\mathrm{O} 2 \mathrm{I}$ and O3 If*. In these cases Martins et al. (2005) determined a $T_{\text {eff }}<50000 \mathrm{~K}$, so perhaps the transition temperature from absorption to emission is somewhat lower than $50000 \mathrm{~K}$.

\section{Summary of conclusions}

The number of CSPNs described as WELS in the literature is 72 . Our spectroscopic survey of 19 WELS stars has shown a variety of spectral types. Of these, we determine that 12 have $\mathrm{H}$ rich atmospheres, with different wind densities. In five cases we could not decide (however, they are not [WR]). Two cases seem to be $\mathrm{H}$-deficient, although even better spectra would be desirable to confirm this.

We find no reason to assume that the WELS denomination is predominantly associated with $\mathrm{H}$ deficiency, and our empirical test indicates that the use of WELS as a spectral type should be discouraged. The WELS denomination should be restricted to mean "insufficient spectral information" to decide if these stars are $\mathrm{H}$ rich or not. Most of the WELS stars, in fact, turn out to be $\mathrm{H}$ rich.

A by-product of this study is that we have found several CSPN spectra with strong, well-defined metal absorption and emission lines that would be suitable targets in a search for spectroscopic binaries.

Acknowledgements. We thank the referee Dr. Klaus Werner whose very useful remarks helped us to improve this paper. This work was primarily based on observations obtained at the Gemini Observatory, which is operated by the Association of Universities for Research in Astronomy, Inc., under a cooperative agreement with the NSF on behalf of the Gemini partnership: the National Science Foundation (US), the Science and Technology Facilities Council (UK), the National Research Council (Canada), CONICYT (Chile), the Australian Research Council (Australia), Ministério da Ciência e Tecnologia (Brazil) and Ministerio de Ciencia, Tecnología e Innovación Productiva (Argentina).

\section{References}

Acker, A., Ochsenbein, F., \& Stenholm, B. 1990, Strasbourg - ESO catalogue of galactic planetary nebulae

Acker, A., Marcout, J., Ochsenbein, F., et al. 1992, The Strasbourg-ESO Catalogue of Galactic Planetary Nebulae, 1047
Aller, L. H., \& Keyes, C. D. 1987, ApJS, 65, 405

Bessell, M. S. 1999, PASP, 111, 1426

Conti, P. S. 1974, ApJ, 187, 539

Corradi, R. L. M., Sabin, L., Miszalski, B., et al. 2011, MNRAS, 410, 1349

De Marco, O., Hillwig, T. C., \& Smith, A. J. 2008, AJ, 136, 323

Drilling, J. S., Jeffery, C. S., Heber, U., Moehler, S., \& Napiwotzki, R. 2013, A\&A, 551, A31

Fogel, J., De Marco, O., \& Jacoby, G. 2003, in Planetary Nebulae: Their Evolution and Role in the Universe, eds. S. Kwok, M. Dopita, \& R. Sutherland, IAU Symp., 209, 235

Gauba, G., Parthasarathy, M., Nakada, Y., \& Fujii, T. 2001, A\&A, 373, 572

Georgiev, L. N., Peimbert, M., Hillier, D. J., et al. 2008, ApJ, 681, 333

Górny, S. K. 2014, A\&A, 570, A26

Górny, S. K., Chiappini, C., Stasińska, G., \& Cuisinier, F. 2009, A\&A, 500, 1089

Handler, G. 2003, in Planetary Nebulae: Their Evolution and Role in the Universe, eds. S. Kwok, M. Dopita, \& R. Sutherland, IAU Symp., 209, 237

Hillwig, T. C., Bond, H. E., \& Afsar, M. 2006, in Planetary Nebulae in our Galaxy and Beyond, eds. M. J. Barlow, \& R. H. Méndez, IAU Symp., 234, 421

Hultzsch, P. J. N., Puls, J., Méndez, R. H., et al. 2007, A\&A, 467, 1253

Iben, Jr., I. 1984, ApJ, 277, 333

Jaschek, C., \& Jaschek, M. 1995, The Behavior of Chemical Elements in Stars (Cambridge, UK: Cambridge University Press), 338

Kwitter, K. B., Méndez, R. H., Peña, M., et al. 2014, Rev. Mex. Astron. Astrofis., 50,203

Marcolino, W. L. F., \& de Araújo, F. X. 2003, AJ, 126, 887

Martins, F., Schaerer, D., \& Hillier, D. J. 2005, A\&A, 436, 1049

Mendez, R. H. 1991, in Evolution of Stars: the Photospheric Abundance Connection, eds. G. Michaud, \& A. V. Tutukov, IAU Symp., 145, 375

Mendez, R. H., \& Niemela, V. S. 1979, ApJ, 232, 496

Mendez, R. H., Herrero, A., \& Manchado, A. 1990, A\&A, 229, 152

Miszalski, B. 2012, in IAU Symp., 283, 107

Schonberner, D. 1989, in Planetary Nebulae, ed. S. Torres-Peimbert, IAU Symp., 131,463

Sota, A., Maíz Apellániz, J., Walborn, N. R., et al. 2011, ApJS, 193, 24

Sota, A., Maíz Apellániz, J., Morrell, N. I., et al. 2014, ApJS, 211, 10

Tylenda, R., Acker, A., \& Stenholm, B. 1993, A\&AS, 102, 595

Walborn, N. 2001, in Eta Carinae and Other Mysterious Stars: The Hidden Opportunities of Emission Spectroscopy, eds. T. R. Gull, S. Johannson, \& K. Davidson, ASP Conf. Ser., 242, 217

Walborn, N. R., \& Howarth, I. D. 2000, PASP, 112, 1446

Walborn, N. R., Sota, A., Maíz Apellániz, J., et al. 2010, ApJ, 711, L143

Weidmann, W. A., \& Gamen, R. 2011a, A\&A, 531, A172

Weidmann, W. A., \& Gamen, R. 2011b, A\&A, 526, A6

Werner, K., \& Herwig, F. 2006, PASP, 118, 183 